Publ. RIMS, Kyoto Univ.

13 (1977), 285-300

\title{
A Class of Approximations of Brownian Motion
}

Dedicated to Professor K. Itô on his 60 th birthday

By

Nobuyuki IKEDA,* Shintaro NAKAO** and Yuiti YAMATO*

\section{$\S 1$. Introduction}

Let $B(t)=\left(B^{1}(t), B^{2}(t), \cdots, B^{d}(t)\right)$ be a $d$-dimensional Brownian motion and let $\left\{B_{n}(t)=\left(B_{n}{ }^{1}(t), B_{n}{ }^{2}(t), \cdots, B_{n}{ }^{d}(t)\right)\right\}$ be a sequence of approximations to $B(t)$. We assume that the sample paths of $B_{n}(t)$ are continuous and piecewise smooth for each $n$ and $B_{n}(t)$ converges to $B(t)$. Let $u(x)$ be a twice continuously differentiable function on $R^{d}$ whose partial derivatives of order $\leqq 2$ are all bounded. In the one-dimensional case E. Wong and M.Zakai [5] showed that $\int_{0}^{t} u\left(B_{n}(s)\right) d B_{n}(s)$ converges to $\int_{0}^{t} u(B(s)) \circ d B(s)$ where the symbol o denotes the symmetric stochastic integral of Stratonovich (K. Itô [2]). They also dealt with the convergence of the more general functional of $B_{n}(\cdot)$, ([6]). In the two-dimensional case P. Lévy [3] showed that $S(t ; n)=\int_{0}^{t}\left(B_{n}{ }^{1}(s)\right.$. $\left.d B_{n}{ }^{2}(s)-B_{n}{ }^{2}(s) d B_{n}{ }^{1}(s)\right) / 2$ converges to the stochastic integral $S(t)=$ $\int_{0}^{t}\left(B^{1}(s) \circ d B^{2}(s)-B^{2}(s) \circ d B^{1}(s)\right) / 2$ if $\left\{B_{n}(t)\right\}$ is a sequence of polygonal approximations to $B(t)$. E.J. McShane [4], on the other hand, gave an example of the sequence $\left\{B_{n}(t)\right\}$ of approximations to $B(t)$ such that $S(t ; n)$ converges to $S(t)+t / \pi$.

In this paper we treat systematically a class of approximations of Brownian motion including McShane's example. In Section 2 we state the main results of the paper. We consider a sequence of Stieltjes integrals of the form $I_{n}(u)=\int_{0}^{t} u\left(B_{n}(s)\right) d B_{n}^{j}(s)$. First we will give some conditions under which $I_{n}(u)$ converges in the quadratic-mean sense. It

Communicated by K. Itô, October 21, 1976.

* Department of Mathematics, Osaka University, Toyonaka 560, Japan.

** Department of Mathematics, Nara Women's University, Nara 630, Japan. 
is then shown that the limit of $I_{n}(u)$ is expressed as the sum of the symmetric stochastic integral $\int_{0}^{t} u(B(s)) \circ d B^{j}(s)$ and a certain "correction term", (cf. Theorem 2.1). In particular, we will give a criterion such that $S(t ; n)$ converges to $S(t)$, (cf. Corollary 2.1 in Section 2). We will also give a couple of examples for Theorem 2.1 in which the correction terms really appear. Section 3 is devoted to the proof of Theorem 2. 1. Finally Section 4 concerns the convergence of the solutions of the ordinary differential equations determined by $B_{n}(t)$.

\section{$\S$ 2. Approximations of Stochastic Integral}

Let $\Omega$ be the space of continuous functions defined on $[0, \infty)$ with values in $R^{d}$. The value of the function $\omega \in \Omega$ at time $t$ will be denoted by $B(t, \omega)=\left(B^{1}(t, \omega), B^{2}(t, \omega), \cdots, B^{d}(t, \omega)\right)$. The argument $\omega$ may be suppressed occasionally. $\mathscr{F}_{t}$ and $\mathscr{F}$ denote the smallest $\sigma$-algebras with respect to which $B(s, \omega)$ are measurable for $0 \leqq s \leqq t$ and for $0 \leqq s<\infty$ respectively. The shift operator is denoted by $\theta_{t}$ : that is $B\left(s, \theta_{t} \omega\right)$ $=B(s+t, \omega),(s \geqq 0)$. Let $\left(\Omega, \mathscr{F}_{1}, \mathscr{F}_{t}, B(t), \theta_{t}, P_{x}\right)$ be the $d$-dimensional Brownian motion. In this paper the following class of the approximations of the Brownian motion will be considered.

Definition 2. 1. Let $\left\{B_{\delta}(t, \omega)=\left(B_{\hat{\delta}}{ }^{1}(t, \omega), B_{\delta}{ }^{2}(t, \omega), \cdots, B_{\delta}{ }^{d}(t, \omega)\right)\right.$; $\delta>0\}$ be a family of $R^{d}$-valued stochastic processes defined on $\left(\Omega, \mathscr{F}, P_{x}\right)$ and let $\kappa$ be a positive constant. We say $\left\{B_{\delta}(t, \omega)\right\} \in \mathcal{A}(B ; \kappa)$ if, for each $\delta>0, B_{\delta}(t, \omega)$ satisfies the following conditions:

(A. 1) $\quad B_{\delta}(k \delta, \omega)=B(k \delta, \omega), \quad$ for $\omega \in \Omega$ and $k=0,1, \cdots$.

(A. 2) $B_{\delta}\left(t+k \delta,(\omega)=B_{\delta}\left(t, \theta_{k \delta}(\omega), \quad\right.\right.$ for $\omega \in \Omega$ and $k=1,2, \cdots$.

(A. 3) $B_{\delta}(t, \omega+x)=B_{\delta}(t, \omega)+x, \quad$ for $\omega \in \Omega, t>0$ and $x \in R^{d}$,

where $\omega+x$ is the function defined by $(\omega+x)(t)=B(t, \omega)+x, t \geqq 0$.

(A. 4) $B_{\delta}(t, \omega)$ is $\mathscr{F}_{\delta}$-measurable for $0 \leqq t \leqq \delta$.

(A. 5) $B_{\delta}(t, \omega)$ is continuous and piecewise smooth in $t$ for $\omega \in \Omega$.

(A. 6) $\quad E_{0}\left[\left(\int_{0}^{\delta}\left|\dot{B}_{\delta}{ }^{i}(s)\right| d s\right)^{6}\right] \leqq \kappa \delta^{3}, \quad$ for $i=1,2, \cdots, d$, 
where $\dot{B}_{\delta}{ }^{i}(s)=\frac{\partial}{\partial s} B_{\delta}{ }^{i}(s), i=1,2, \cdots, d$, and $E_{x}[\cdot]$ denotes the expectation with respect to the probability measure $P_{x}$.

Let us consider a differential 1-form on $R^{d}$ of the following form:

$$
\alpha_{i j}=\left(x^{i} d x^{j}-x^{j} d x^{i}\right) / 2, \quad(i, j=1,2, \cdots, d),
$$

and let $S_{i j}(t ; \delta)$ be the integral of $\alpha_{i j}$ along $C_{\delta}[0, t]$ : i.e.

$$
S_{i j}(t ; \delta)=\int_{C_{j}[0, t]} \alpha_{i j}
$$

where $C_{\delta}[0, \iota]$ is the curve defined by $C_{\delta}[0, \mathrm{t}]=\left\{B_{\delta}(s, \omega) ; 0 \leqq s \leqq t\right\}$. Then we have

$$
S_{i j}(t ; \delta)=\int_{0}^{t}\left(B_{\delta}{ }^{i}(s) d B_{\delta}{ }^{j}(s)-B_{\delta}{ }^{j}(s) d B_{\delta}{ }^{i}(s)\right) / 2 .
$$

Setting

$$
s_{i j}(\delta)=E_{0}\left[S_{i j}(\delta ; \delta)\right] / \delta,
$$

we have

Proposition 2.1. Suppose $\left\{B_{\delta}(t)\right\} \in \mathcal{A}(B ; \kappa)$ for some positive constant $\kappa$. Then there exists a sequence $\left\{\delta_{n}\right\}$ of positive numbers such that $\lim _{n \rightarrow \infty} \delta_{n}=0$ and for $1 \leqq i, j \leqq d$ the sequence $\left\{s_{i j}\left(\delta_{n}\right)\right\}$ has a finite limit as $n \rightarrow \infty$.

Proof. Fix $i$ and $j$. To complete the proof we need only to show that $\left\{s_{i j}(0)\right\}$ is bounded. By (A. 6), we have

$$
\begin{aligned}
\left|s_{i j}(\delta)\right| & \leqq E_{0}\left[\int_{0}^{\grave{o}}\left|\dot{B}_{\delta}{ }^{i}(s)\right| d s \int_{0}^{\delta}\left|\dot{B}_{\delta}^{j}(s)\right| d s\right] / \delta \\
& \leqq\left(E_{0}\left[\left(\int_{0}^{\delta}\left|\dot{B}_{\delta}^{i}(s)\right| d s\right)^{6}\right]\right)^{1 / 6}\left(E_{0}\left[\left(\int_{0}^{o}\left|\dot{B}_{\delta}^{j}(s)\right| d s\right)^{6}\right]\right)^{1 / 6} / \delta \\
& \leqq \kappa^{1 / 3} .
\end{aligned}
$$

This estimate proves the proposition.

In the remainder of the paper let $S=\left(s_{i j}\right),(1 \leqq i, j \leqq d)$, be a skewsymmetric $d \times d$-matrix and let $\left\{\delta_{n}\right\}$ be a sequence of positive numbers 
satisfying $\lim _{n \rightarrow \infty} \delta_{n}=0$. Now we will give a notation.

Definition 2.2. Let $\left\{B_{\delta}(t)\right\} \in \mathcal{A}(B ; \kappa)$. We say $\left\{B_{\delta_{n}}(t)\right\} \in \mathcal{A}(B$; $\kappa, S)$ if

$$
\lim _{n \rightarrow \infty} s_{i j}\left(\delta_{n}\right)=s_{i j}, \quad \text { for every } \quad 1 \leqq i, j \leqq d
$$

Still some more notation is needed. Let $\mathcal{H}\left(R^{d}\right)$ be the space of twice continuously differentiable functions on $R^{d}$ whose partial derivatives of order $\leqq 2$ are all bounded. Finally set

$$
\begin{array}{r}
S_{i j}(t)=\int_{0}^{t}\left(B^{i}(s) \circ d B^{j}(s)-B^{j}(s) \circ d B^{i}(s)\right) / 2, \quad t>0, \\
i, j=1,2, \cdots, d .
\end{array}
$$

The result we want to show is the following:

Theorem 2.1. Suppose $\left\{B_{\delta}(t)\right\} \in \mathcal{A}(B ; \kappa)$ for some positive constant $\kappa$. Let $S=\left(s_{i j}\right)$ be a skere-symmetric $d \times d$-matrix. Then the following four statements are equivalent.

$$
\begin{array}{ll}
\text { (i) } & \left\{B_{\hat{v}_{n}}(t)\right\} \in \mathcal{A}(B ; \kappa, S) . \\
\text { (ii) } & \lim _{n \rightarrow \infty} E_{0}\left[\left|S_{i j}\left(t ; \delta_{n}\right)-S_{i j}(t)-s_{i j} t\right|^{2}\right]=0,
\end{array}
$$

$$
\text { for } 1 \leqq i, j \leqq d \text { and } t>0 .
$$

(iii) $\quad \lim _{n \rightarrow \infty} E_{0}\left[\left|\int_{0}^{t} B_{\delta_{n}}^{i}(s) d B_{\delta_{n}}^{j}(s)-\int_{0}^{t} B^{i}(s) \circ d B^{j}(s)-s_{i j} t\right|^{2}\right]=0$,

$$
\text { for } 1 \leqq i, j \leqq d \text { and } t>0 \text {. }
$$

(iv) $\quad \lim _{n \rightarrow \infty} E_{0}\left[\mid \int_{0}^{t} u\left(B_{\delta_{n}}(s)\right) d B_{\delta_{n}}^{j}(s)-\int_{0}^{t} u(B(s)) \circ d B^{j}(s)\right.$

$$
\begin{aligned}
& \left.-\left.\sum_{i=1}^{d} s_{i j} \int_{0}^{t} \frac{\partial}{\partial x^{i}} u(B(s)) d s\right|^{2}\right]=0, \\
& \quad \text { for } u \in \mathcal{H}\left(R^{d}\right), 1 \leqq j \leqq d \text { and } t>0 .
\end{aligned}
$$

The proof of Theorem 2.1 will be given in the next section. Now we will define a typical subclass of approximations in $\mathcal{A}(B ; \kappa, S)$. 
Definition 2.3. Let $\left\{B_{\widehat{o}_{n}}(t)\right\} \in \mathcal{A}(B ; \kappa, S)$. We say that $\left\{B_{\hat{o}_{n}}(I)\right\}$ is symmetric if each component of $S$ is equal to 0 .

The following corollary is an immediate consequence of Theorem 2. 1 .

Corollary 2.1. Let $\left\{B_{\delta_{n}}(t)\right\} \in \mathcal{A}(B ; \kappa, S)$. Then $\left\{B_{\delta_{n}}(t)\right\}$ is symmetric if and only if

$$
\begin{aligned}
& \lim _{n \rightarrow \infty} E_{0}\left[\left|S_{i j}\left(t ; \hat{o}_{n}\right)-S_{i j}(t)\right|^{2}\right]=0 \\
& \qquad \text { for } 1 \leqq i, j \leqq d \text { and } t>0
\end{aligned}
$$

Remark 2.1. Let $B(t)=\left(B^{1}(t), B^{2}(t)\right)$ be a two-dimensional Brownian motion starting at 0 and let $C_{\delta}{ }^{*}=\left\{C_{\delta}^{*}(s) ; 0 \leqq s \leqq t+1\right\}$ be the closed curve in $R^{2}$ defined by

$$
C_{\delta}{ }^{*}(s)= \begin{cases}\left(B_{\delta}{ }^{1}(s), B_{\delta}{ }^{2}(s)\right), & 0 \leqq s \leqq t, \\ (t+1-s)\left(B_{\delta}{ }^{1}(t), B_{o}{ }^{2}(t)\right), & t<s \leqq t+1 .\end{cases}
$$

As mentioned in the Introduction, P. Lévy [3] proved (2.3) in the case that $\left\{B_{\delta_{n}}(t)\right\}$ is a sequence of polygonal approximations to $B(t)$. In this case, we can write

$$
S_{12}(t ; \delta)=\int_{{C_{\hat{\sigma}}}^{*}} \alpha
$$

where $\alpha=\left(x^{1} d x^{2}-x^{2} d x^{1}\right) / 2$. We may, therefore, consider $S_{12}(t)$ as a stochastically defined area enclosed by a Brownian curve up to moment $t$ and its chord, (P. Lévy [3], pp. 262-266).

Remark 2. 2. Suppose $\left\{B_{\delta_{n}}(t)\right\} \in \mathcal{A}(B ; \kappa, S)$. If $\left\{B_{\delta_{n}}(t)\right\}$ is symmetric, then $\left(B_{\delta_{n}}^{i}(t), S_{j k}\left(t ; \delta_{n}\right)\right),(1 \leqq i, j, k \leqq d)$, converges to the diffusion process $\left(B^{i}(t), S_{j k}(t)\right),(1 \leqq i, j, k \leqq d)$, in $L^{2}\left(\Omega, P_{0}\right)$, (cf. M.B. Gaveau [1]).

Finally we will give three examples. For this purpose we introduce the following notations. $\Phi$ denotes the space of continuously differentiable functions $\phi(t)$ on $[0,1]$ such that

$$
\phi(0)=0 \quad \text { and } \quad \phi(1)=1 \text {. }
$$


For $\phi \in \Phi$, set $\dot{\phi}=\frac{d}{d t} \phi$. For $\delta>0$ and $k=0,1, \cdots$, set

$$
\Delta_{k} B^{i}=B^{i}(k \delta+\delta)-B^{i}(k \delta) .
$$

Example 2. 1. Let $\phi^{k} \in \Phi, k=1,2, \cdots, d$. Set, for $i=1,2, \cdots, d$,

$$
B_{\delta}{ }^{i}(t)=B^{i}(k \delta)+\phi^{i}((t-k \delta) / \delta) \Delta_{k} B^{i}, \quad \text { if } k \delta \leqq t<k \delta+\delta, k=0,1, \cdots .
$$

Then $\left\{B_{\delta}(t)=\left(B_{\delta}{ }^{1}(t), B_{\delta}{ }^{2}(t), \cdots, B_{\delta}{ }^{d}(t)\right)\right\} \in \mathcal{A}(B ; \kappa)$ for some positive constant $\kappa$. In this case, since

$$
s_{i j}(\delta)=0, \quad \text { for every } \delta>0 \text { and } 1 \leqq i, j \leqq d,
$$

$\left\{B_{\delta}(t)\right\}$ is symmetric. Hence if $\left\{B_{\hat{\delta}}(t)\right\}$ is a sequence of polygonal approximations to $B(t),\left\{B_{\delta}(t)\right\}$ is symmetric and $S_{i j}(t ; \delta)$ converges to $S_{i j}(t)$ in the quadratic-mean sense.

Example 2. 2. (E.J. McShane [4]). Let $d=2$ and let $\phi^{i} \in \emptyset, i=1,2$. For $i=1,2$, we define

(2.4) $\quad B_{\delta}^{i}(t)=\left\{\begin{array}{rrr}B^{i}(k \delta)+\phi^{i}((t-k \delta) / \delta) \Delta_{k} B^{i}, & \text { if } \Delta_{k} B^{1} \Delta_{k} B^{2} \geqq 0, \\ B^{i}(k \delta)+\phi^{3-i}((t-k \delta) / \delta) \Delta_{k} B^{i}, & \text { if } \Delta_{k} B^{1} \Delta_{k} B^{2}<0,\end{array}\right.$

Then $\left\{B_{\delta}(t)=\left(B_{\delta}{ }^{1}(t), B_{\delta}{ }^{2}(t)\right)\right\} \in \mathcal{A}(B ; \kappa)$ for some positive constant $\kappa$. By (2.1) and (2.4) we have

$$
\begin{aligned}
S_{12}(\delta ; \delta)=\frac{\left|\Delta_{0} B^{1} \Delta_{0} B^{2}\right|}{2} & \left\{1-2 \int_{0}^{1} \dot{\phi}^{1}(s) \phi^{2}(s) d s\right\} \\
+ & {\left[B^{1}(0) B^{2}(\delta)-B^{2}(0) B^{1}(\delta)\right] / 2 . }
\end{aligned}
$$

Since $E_{0}\left[\left|\Delta_{0} B^{1} \Delta_{0} B^{2}\right|\right]=2 \delta / \pi$, it follows that

$$
s_{12}(\delta)=\left(1-2 \int_{0}^{1} \dot{\phi}^{1}(s) \phi^{2}(s) d s\right) / \pi, \quad \text { for every } \delta>0 .
$$

Example 2. 3. Let $\phi_{j}{ }^{i} \in \Phi, \quad(i=1,2, \cdots, d$ and $j=1,2)$. Set, for $i=1,2, \cdots, d$,

(2.5) $\quad B_{\delta}{ }^{i}(t)=\left\{\begin{array}{cc}B^{i}(k \delta)+\phi_{1}{ }^{i}((t-k \delta) / \delta) \Delta_{k} B^{i}, & \text { if } \Delta_{k} B^{i} \geqq 0, \\ B^{i}(k \delta)+\phi_{2}{ }^{i}((t-k \delta) / \delta) \Delta_{k} B^{i}, & \text { if } \Delta_{k} B^{i}<0,\end{array}\right.$ 
Then $\left\{B_{\delta}(t)=\left(B_{\delta}{ }^{1}(t), B_{\delta}{ }^{2}(t), \cdots, B_{\delta}{ }^{d}(t)\right)\right\} \in \mathcal{A}(B ; \kappa)$ for some positive constant $\kappa$. By (2.1) and (2.5), we have, for every $\delta>0$ and $i \neq j$, $S_{i j}^{*}(\delta ; \delta)$

$$
= \begin{cases}\left|\Delta_{0} B^{i} \Delta_{0} B^{j}\right|\left(1-2 \int_{0}^{1} \dot{\phi}_{1}^{i}(s) \phi_{1}^{j}(s) d s\right) / 2, & \text { if } \Delta_{0} B^{i} \geqq 0, \Delta_{0} B^{j} \geqq 0, \\ -\left|\Delta_{0} B^{i} \Delta_{0} B^{j}\right|\left(1-2 \int_{0}^{1} \dot{\phi}_{1}{ }^{i}(s) \phi_{2}{ }^{j}(s) d s\right) / 2, & \text { if } \Delta_{0} B^{i} \geqq 0, \Delta_{0} B^{j}<0, \\ -\left|\Delta_{0} B^{i} \Delta_{0} B^{j}\right|\left(1-2 \int_{0}^{1} \dot{\phi}_{2}{ }^{i}(s) \phi_{1}^{j}(s) d s\right) / 2, & \text { if } \Delta_{0} B^{i}<0, \Delta_{0} B^{j} \geqq 0, \\ \left|\Delta_{0} B^{i} \Delta_{0} B^{j}\right|\left(1-2 \int_{0}^{1} \dot{\phi}_{2}{ }^{i}(s) \phi_{2}{ }^{j}(s) d s\right) / 2, & \text { if } \Delta_{0} B^{i}<0, \Delta_{0} B^{j}<0,\end{cases}
$$

where $S_{i j}^{*}(\delta ; \delta)=S_{i j}(\delta ; \delta)-\left[B^{i}(0) B^{j}(\delta)-B^{j}(0) B^{i}(\delta)\right] / 2$. Hence

$$
\begin{aligned}
s_{i j}(\delta)=-\int_{0}^{1}\left(\dot{\phi}_{1}{ }^{i}-\dot{\phi}_{2}{ }^{i}\right)(s)\left(\phi_{1}{ }^{j}-\phi_{2}{ }^{j}\right)(s) d s / 2 \pi, \\
\text { for every } \delta>0 \text { and } i \neq j .
\end{aligned}
$$

Using (2.6) we can prove that for any skew-symmetric $d \times d$-matrix $S$, there exists a sequence $\left\{B_{\hat{o}_{n}}(t)\right\}$ of approximations to $B(t)$ such that $\left\{B_{\delta_{n}}(t)\right\} \in \mathcal{A}(B ; \kappa, S)$.

\section{§ 3. Proof of Theorem 2.1}

Before proceeding to the proof of Theorem 2.1 we will prepare four lemmas. Set

$$
c_{i j}(\delta)=E_{0}\left[\int_{0}^{\hat{o}} \dot{B}_{\delta}{ }^{i}(s)\left(B_{\delta}{ }^{j}(\delta)-B_{\delta}{ }^{j}(s)\right) d s\right] / \delta .
$$

Lemma 3. 1. For $\delta>0$,

$$
\begin{array}{ll}
c_{i i}(\delta)=1 / 2, & \text { for } 1 \leqq i \leqq d, \\
c_{i j}(\delta)=s_{i j}(\delta), & \text { for } 1 \leqq i, j \leqq d \text { and } i \neq j .
\end{array}
$$

Proof. By (3.1),

$$
c_{i j}(\delta)+c_{j i}(\delta)=E_{0}\left[B^{i}(\delta) B^{j}(\delta)\right] / \delta .
$$

Since $E_{0}\left[B^{i}(\delta) B^{j}(\delta)\right]=\delta \delta_{i, j}$, we have 


$$
c_{i i}(\delta)=1 / 2 \text { and } c_{i j}(\delta)=-c_{j i}(\delta) \quad \text { for } i \neq \mathrm{j} .
$$

Combining this with (3.1) we can prove that if $i \neq j$, then

$$
\begin{aligned}
c_{i j}(\delta) & =\left(c_{i j}(\delta)-c_{j i}(\delta)\right) / 2 \\
& =E_{0}\left[\int_{0}^{\delta}\left(B_{\delta}{ }^{i}(s) d B_{\delta}{ }^{j}(s)-B_{\delta}{ }^{j}(s) d B_{\delta}{ }^{i}(s)\right)\right] / 2 \delta \\
& =s_{i j}(\delta) .
\end{aligned}
$$

This completes the proof of Lemma 3.1.

Lemma 3. 2. For any $\delta>0$ and $1 \leqq i, j \leqq d$,

$$
\begin{array}{r}
E_{x}\left[\left\{\int_{0}^{\delta} \dot{B}_{\delta}^{i}(s)\left(B_{\delta}^{j}(\delta)-B_{\delta}^{j}(s)\right) d s\right\}^{p}\right] \\
=E_{0}\left[\left\{\int_{0}^{o} \dot{B}_{\delta}^{i}(s)\left(B_{\delta}^{j}(\delta)-B_{\delta}{ }^{j}(s)\right) d s\right\}^{p}\right], \\
\text { for } p=1,2 \text { and } x \in R^{d},
\end{array}
$$

and

$$
\begin{array}{r}
E_{0}\left[\int_{k \delta}^{(k+1) \delta} \dot{B}_{\delta}{ }^{i}(s)\left(B_{\delta}{ }^{j}(k \delta+\delta)-B_{\delta}{ }^{j}(s)\right) d s / \mathscr{F}_{k \delta}\right] \\
\text { for } k=0 c_{i j}(\delta),
\end{array}
$$

Proof. (3.5) follows from (A. 3). Appealing to the Markov property, we have

$$
\begin{gathered}
E_{0}\left[\int_{k \delta}^{(k+1) \delta} \dot{B}_{\delta}^{i}(s)\left(B_{\delta}^{j}(k \delta+\delta)-B_{\delta}^{j}(s)\right) d s / \mathscr{F}_{k \delta}\right] \\
=E_{0}\left[\int _ { 0 } ^ { \delta } \dot { B } _ { \delta } ^ { i } \left(s, \theta_{k \delta}(\omega)\left(B_{\delta}{ }^{j}\left(\delta, \theta_{k \delta}(\omega)-B_{\delta}{ }^{j}\left(s, \theta_{k \delta}(\omega)\right) d s / \mathscr{F}_{k \delta}\right],\right.\right.\right. \\
\quad \text { (by (A. 2)), } \\
=E_{B(k \delta)}\left[\int_{0}^{\delta} \dot{B}_{\delta}^{i}(s)\left(B_{\delta}{ }^{j}(\delta)-B_{\delta}{ }^{j}(s)\right) d s\right] .
\end{gathered}
$$

Combining this with (3.5) we can complete the proof of Lemma 3.2.

For the sake of brevity, we introduce the following notations. For $\delta>0$, set 


$$
\left\{\begin{array}{l}
{[s]^{+}(\delta)=(k+1) \delta} \\
{[s]^{-}(\delta)=k \delta}
\end{array} \quad, \text { for } k \delta \leqq s<(k+1) \delta, \quad(k=0,1,2, \cdots)\right.
$$

Setting $s(\delta)=[s]^{-}(\delta) / \delta$, we have

Lemma 3. 3. Let $Z_{1}(s, \omega)$ be a bounded $\mathscr{F}_{s}$-adapted process defined on $\left(\Omega, \mathscr{I}, P_{x}\right)$ rvith piecewise continuous sample paths. If $\left\{B_{\delta}(t)\right\} \in \mathscr{A}(B ; \kappa)$, then

$$
\begin{gathered}
E_{0}\left[\left\{\int_{0}^{[t]^{(\delta)}} Z_{1}\left([s]^{-}(\delta)\right)\left[\dot{B}_{\delta}{ }^{i}(s)\left(B_{\delta}{ }^{j}\left([s]^{+}(\delta)\right)-B_{\delta}{ }^{j}(s)\right)-c_{i j}(\delta)\right] d s\right\}^{2}\right] \\
\leqq \kappa^{2 / 3}\left(K_{1}\right)^{2}[t]^{-}(\delta) \delta, \quad \text { for } 1 \leqq i, j \leqq d \text { and } t>0,
\end{gathered}
$$

where $K_{1}=\sup _{s, \omega}\left|Z_{1}(s, \omega)\right|$.

Proof. Since

$$
E_{0}\left[\int_{k \grave{ }}^{(k+1) \grave{\delta}}\left[\dot{B}_{\delta}{ }^{i}(s)\left(B_{\delta}{ }^{j}(k \delta+\delta)-B_{\delta}{ }^{j}(s)\right)-c_{i j}(\delta)\right] d s / \mathscr{F}_{k \delta}\right]=0
$$

from (3.6), it follows that

$$
\begin{array}{r}
E_{0}\left[\left\{\int _ { 0 } ^ { [ t ] ^ { - ( \delta ) } } Z _ { 1 } ( [ s ] ^ { - } ( \delta ) ) \left[\dot{B}_{\delta}{ }^{i}(s)\left(B_{\delta}{ }^{j}\left([s]^{+}(\delta)\right)-B_{\delta}{ }^{j}(s)\right)\right.\right.\right. \\
\left.\left.\left.-c_{i j}(\delta)\right] d s\right\}^{2}\right] \\
=E_{0}\left[\sum _ { k = 0 } ^ { t ( \delta ) - 1 } Z _ { 1 } ( k \delta ) ^ { 2 } \left\{\int _ { k \delta } ^ { ( k + 1 ) \delta } \left[\dot{B}_{\delta}^{i}(s)\left(B_{\delta}^{j}(k \delta+\delta)-B_{\delta}^{j}(s)\right)\right.\right.\right. \\
\left.\left.\left.-c_{i j}(\delta)\right] d s\right\}^{2}\right] .
\end{array}
$$

Using Lemma 3. 2, we have

$$
\begin{array}{r}
E_{0}\left[\left(\int_{k \delta}^{(k+1) \delta}\left[\dot{B}_{\delta}{ }^{i}(s)\left(B_{\delta}{ }^{j}(k \delta+\delta)-B_{\delta}{ }^{j}(s)\right)-c_{i j}(\delta)\right] d s\right)^{2} / \mathscr{F}_{k \delta}\right] \\
=E_{0}\left[\left(\int_{0}^{\grave{o}} \dot{B}_{\delta}{ }^{i}(s)\left(B_{\delta}{ }^{j}(\delta)-B_{\delta}{ }^{j}(s)\right) d s\right)^{2}\right]-\left(c_{i j}(\delta) \delta\right)^{2} .
\end{array}
$$

On the other hand, by (A.6) in Section 2,

$$
E_{0}\left[\left(\int_{0}^{\delta} \dot{B}_{\delta}^{i}(s)\left(B_{\delta}{ }^{j}(\delta)-B_{\delta}{ }^{j}(s)\right) d s\right)^{2}\right]
$$




$$
\begin{aligned}
& \leqq E_{0}\left[\left(\int_{0}^{\delta}\left|\dot{B}_{\delta}{ }^{i}(s)\right| d s\right)^{2}\left(\int_{0}^{\delta}\left|\dot{B}_{\delta}{ }^{j}(s)\right| d s\right)^{2}\right] \\
& \leqq \kappa^{2 / 3} \delta^{2} .
\end{aligned}
$$

Combining (3.7), (3.8) and (3.9), we have

$$
\begin{aligned}
& E_{0}\left[\left\{\int_{0}^{[t]^{-(\delta)}} Z_{1}\left([s]^{-}(\delta)\right)\left[\dot{B}_{\delta}^{i}(s)\left(B_{\delta}^{j}\left([s]^{+}(\delta)\right)-B_{\delta}^{j}(s)\right)-c_{i j}(\delta)\right] d s\right\}^{2}\right] \\
& \quad \leqq \kappa^{2 / 3}\left(K_{1}\right)^{2}[t]^{-}(\delta) \delta
\end{aligned}
$$

which completes the proof of Lemma 3.3.

Lemma 3. 4. Let $K_{2}$ be a positive constant and let $Z_{2}(s, \omega)$ be a stochastic process defined on $\left(\Omega, \mathscr{F}, P_{x}\right.$ ) with piecewise continuous sample paths satisfying the following condition:

$$
\left|Z_{2}(s)\right| \leqq K_{2} \sum_{m=1}^{d} \int_{[s]^{-(\delta)}}^{[s]^{+(\delta)}}\left|\dot{B}_{\delta}^{m}(u)\right| d u, \quad \text { for } s \geqq 0 .
$$

If $\left\{B_{\delta}(t)\right\} \in \mathcal{A}(B ; \kappa)$, then

$$
\begin{aligned}
& E_{0}\left[\left\{\int_{0}^{[t]^{-(\delta)}} Z_{2}(s) \dot{B}_{\delta}^{i}(s)\left(B_{\delta}^{j}\left([s]^{+}(\delta)\right)-B_{\delta}{ }^{j}(s)\right) d s\right\}^{2}\right] \\
& \quad \leqq \kappa\left(K_{2}[t]^{-}(\delta) d\right)^{2} \delta, \quad \text { for } 1 \leqq i, j \leqq d \text { and } t>0 .
\end{aligned}
$$

Proof. By (3. 10),

$$
\begin{aligned}
& \left|\int_{0}^{[t]^{-(\delta)}} Z_{2}(s) \dot{B}_{\delta}{ }^{i}(s)\left(B_{\delta}{ }^{j}\left([s]^{+}(\delta)\right)-B_{\delta}{ }^{j}(s)\right) d s\right| \\
& \quad \leqq\left|\sum_{k=0}^{t(\delta)-1} \int_{k \delta}^{(k+1) \delta} Z_{2}(s) \dot{B}_{\delta}{ }^{i}(s)\left(B_{\delta}{ }^{j}\left([s]^{+}(\delta)\right)-B_{\delta}{ }^{j}(s)\right) d s\right| \\
& \quad \leqq K_{2} \sum_{k=0}^{t(\delta)-1} \sum_{m=1}^{d} \int_{k \grave{\delta}}^{(k+1) \delta}\left|\dot{B}_{\delta}{ }^{m}(s)\right| d s \int_{k \grave{\delta}}^{(k+1) \delta}\left|\dot{B}_{\delta}{ }^{i}(s)\right| d s \int_{k \bar{\delta}}^{(k+1) \delta}\left|\dot{B}_{\delta}^{j}(s)\right| d s .
\end{aligned}
$$

Hence by (A. 2), (A. 4) and (A.6), the left-hand side of (3.11) is bounded above by

$$
\begin{aligned}
& \left(K_{2} t(\delta)\right)^{2} \sum_{m=1}^{d} \sum_{k=1}^{d} E_{0}\left[\int_{0}^{\delta}\left|\dot{B}_{\delta}{ }^{m}(s)\right| d s \int_{0}^{\delta}\left|\dot{B}_{\delta}{ }^{k}(s)\right| d s\right. \\
& \left.\quad \times\left(\int_{0}^{\delta}\left|\dot{B}_{\delta}{ }^{i}(s)\right| d s\right)^{2}\left(\int_{0}^{\delta}\left|\dot{B}_{\delta}{ }^{j}(s)\right| d s\right)^{2}\right] \leqq \kappa\left(K_{2}[t]^{-}(\delta) d\right)^{2} \delta,
\end{aligned}
$$

which completes the proof of Lemma 3. 4 . 
We now turn to the proof of Theorem 2.1.

Proof of Theorem 2.1. The implication (iv) $\rightarrow$ (iii) is trivial. Since $s_{i j}=-s_{j i}$, certainly (iii) implies (ii).

Proof of (ii) $\rightarrow$ (i). Suppose (ii) holds. First we note that $s_{i i}(\delta)$ $=0$ for any $\delta>0$. Fix $i$ and $j$ such that $i \neq j$. From (ii), $E_{0}\left[S_{i j}\left(1 ; \delta_{n}\right)\right]$ converges to $s_{i j}$. Hence, using $B_{\delta}(t) \in \mathcal{A}(B ; \kappa)$, we can prove that $E_{0}\left[S_{i j}\left([1]^{-}\left(\delta_{n}\right), \delta_{n}\right)\right]$ converges to $s_{i j}$. Consequently we have

$$
\begin{aligned}
\lim _{n \rightarrow \infty} E_{0}\left[\int _ { 0 } ^ { [ 1 ] - ( \delta _ { n } ) } \left\{\left({B_{\delta_{n}}}^{i}(s)-B_{\delta_{n}}{ }^{i}\left([s]^{+}\left(\delta_{n}\right)\right)\right){\dot{B_{\delta_{n}}}}^{j}(s)\right.\right. \\
\left.\left.\quad-\left({B_{\delta_{n}}}^{j}(s)-{B_{\delta_{n}}}^{j}\left([s]^{+}\left(\delta_{n}\right)\right)\right){\dot{B_{\delta_{n}}}}^{i}(s)\right\} d s\right] / 2=s_{i j} .
\end{aligned}
$$

On the other hand, by Lemma 3.2, the left-hand side of (3.12) is equal to

$$
\begin{aligned}
\lim _{n \rightarrow \infty} \sum_{k=0}^{1\left(\delta_{n}\right)-1} E_{0}\left[\int_{k \delta_{n}}^{(k+1) \delta_{n}}\left(B_{\delta_{n}}{ }^{i}(s)-{B_{\delta_{n}}}^{i}\left((k+1) \delta_{n}\right)\right) \dot{B}_{\delta_{n}}{ }^{j}(s) d s\right. \\
\left.-\int_{k \delta_{n}}^{(k+1) \delta_{n}}\left({B_{\delta_{n}}}^{j}(s)-{B_{\delta_{n}}}^{j}\left((k+1) \delta_{n}\right)\right) \dot{B}_{\delta_{n}}{ }^{i}(s) d s\right] / 2 \\
=\lim _{n \rightarrow \infty} \sum_{k=0}^{1\left(\hat{\delta}_{n}\right)-1} E_{0}\left[\int_{0}^{\delta_{n}}\left({B_{\delta_{n}}}^{j}\left(\delta_{n}\right)-{B_{\delta_{n}}}^{j}(s)\right) \dot{B}_{\delta_{n}}{ }^{i}(s) d s\right. \\
\left.\quad-\int_{0}^{\delta_{n}}\left({B_{\delta_{n}}}^{i}\left(\delta_{n}\right)-{B_{\delta_{n}}}^{i}(s)\right){\dot{B_{\delta_{n}}}}^{j}(s) d s\right] / 2 \\
=\lim _{n \rightarrow \infty}[1]-\left(\delta_{n}\right) c_{i j}\left(\delta_{n}\right) .
\end{aligned}
$$

Hence, by (3.12) and Lemma 3.1,

$$
\lim _{n \rightarrow \infty}[1]^{-}\left(\delta_{n}\right) s_{i j}\left(\delta_{n}\right)=s_{i j},
$$

and (i) follows.

Proof of (i) $\rightarrow$ (iv). Suppose (i) holds. Set $u_{i}=\frac{\partial}{\partial x^{i}} u$ for $u \in$ $\mathcal{H}\left(R^{d}\right)$ and put $c_{i j}=s_{i j}+\delta_{i, j} / 2$. Since

$$
\int_{0}^{t} u(B(s)) \circ d B^{j}(s)=\int_{0}^{t} u(B(s)) d B^{j}(s)+\frac{1}{2} \int_{0}^{t} u_{j}(B(s)) d s,
$$


we have

$$
\begin{aligned}
\int_{0}^{t} u & (B(s)) \circ d B^{j}(s)+\sum_{i=1}^{d} s_{i j} \int_{0}^{t} u_{i}(B(s)) d s \\
= & \int_{0}^{t} u(B(s)) d B^{j}(s)+\sum_{i=1}^{d} c_{i j} \int_{0}^{t} u_{i}(B(s)) d s .
\end{aligned}
$$

By integration by parts, we obtain

$$
\begin{aligned}
\int_{k \delta}^{(k+1) \delta} u\left(B_{\delta}(s)\right) d B_{\delta}^{j}(s) \\
=-\int_{k \delta}^{(k+1) \delta} u\left(B_{\delta}(s)\right) \frac{d}{d s}\left(B_{\delta}^{j}(k \delta+\delta)-B_{\delta}^{j}(s)\right) d s \\
=u\left(B_{\delta}(k \delta)\right)\left(B_{\delta}^{j}(k \delta+\delta)-B_{\delta}^{j}(k \delta)\right) \\
\quad+\sum_{i=1}^{d} \int_{k \delta}^{(k+1) \delta} u_{i}\left(B_{\delta}(s)\right) \dot{B}_{\delta}^{i}(s)\left(B_{\delta}^{j}(k \delta+\delta)-B_{\delta}^{j}(s)\right) d s \\
=u(B(k \delta))\left(B^{j}(k \delta+\delta)-B^{j}(k \delta)\right) \\
\quad+\sum_{i=1}^{d} \int_{k \delta}^{(k+1) \delta} u_{i}\left(B_{\delta}(s)\right) \dot{B}_{\delta}^{i}(s)\left(B_{\delta}^{j}(k \delta+\delta)-B_{\delta}^{j}(s)\right) d s
\end{aligned}
$$

(by (A. 1)).

Now we put

$$
\begin{aligned}
& I_{1}(\delta)=\int_{[t]^{-(\delta)}}^{t} u\left(B_{\delta}(s)\right) d B_{\delta}^{j}(s)-\int_{[t]^{-(\delta)}}^{t} u(B(s)) d B^{j}(s) \\
& -\sum_{i=1}^{d} c_{i j} \int_{[t]^{-(\delta)}}^{t} u_{i}(B(s)) d s \\
& I_{2}(\delta)=\int_{0}^{[t]^{-(\delta)}}\left(u\left(B\left([s]^{-}(\delta)\right)\right)-u(B(s))\right) d B^{j}(s), \\
& I_{3}(\delta)=\sum_{i=1}^{d} \int_{0}^{[t]^{-(\delta)}} u_{i}\left(B\left([s]^{-}(\delta)\right)\right)\left[\dot{B}_{\delta}{ }^{i}(s)\left(B_{\delta}^{j}\left([s]^{+}(\delta)\right)-B_{\delta}^{j}(s)\right)\right. \\
& \left.-c_{i j}(\delta)\right] d s, \\
& I_{4}(\delta)=\sum_{i=1}^{d} \int_{0}^{[t]^{-(\delta)}}\left[u_{i}\left(B_{\delta}(s)\right)-u_{i}\left(B\left([s]^{-}(\delta)\right)\right)\right] \dot{B}_{\delta}{ }^{i}(s)\left(B_{\delta}^{j}\left([s]^{+}(\delta)\right)\right. \\
& \left.-B_{\delta}^{j}(s)\right) d s, \\
& I_{5}(\delta)=\sum_{i=1}^{d} \int_{0}^{[t]^{-(\delta)}}\left[u_{i}\left(B\left([s]^{-}(\delta)\right)\right)-u_{i}(B(s))\right] d s c_{i j},
\end{aligned}
$$




$$
I_{6}(\delta)=\sum_{i=1}^{d} \int_{0}^{[t]^{-(\delta)}} u_{i}\left(B\left([s]^{-}(\hat{\delta})\right)\right) d s\left(c_{i j}(\delta)-c_{i j}\right)
$$

Combining (3.13) with (3.14), we have

$$
\begin{array}{r}
\int_{0}^{t} u\left(B_{\delta}(s)\right) d B_{\delta}{ }^{j}(s)-\int_{0}^{t} u(B(s)) \circ d B^{j}(s) \\
-\sum_{i=1}^{d} s_{i j} \int_{0}^{t} u_{i}(B(s)) d s=\sum_{i=1}^{6} I_{i}(\delta) .
\end{array}
$$

It is obvious that

$$
\lim _{\delta \rightarrow 0} E_{0}\left[\left\{I_{1}(\delta)+I_{2}(\delta)+I_{5}(\delta)\right\}^{2}\right]=0
$$

Applying Lemmas 3.3 and 3.4 to $I_{3}(\delta)$ and $I_{4}(\delta)$ respectively, we have

$$
\lim _{\delta \rightarrow 0} E_{n}\left[\left\{I_{3}(\delta)+I_{1}(\delta)\right\}^{2}\right]=0 .
$$

It is also clear that (i) implies

$$
\lim _{n \rightarrow \infty} E_{0}\left[\left(I_{6}\left(\delta_{n}\right)\right)^{2}\right]=0 .
$$

Combining (3.15), (3.16), (3.17) and (3.18), we can see that (iv) follows from (i).

\section{§4. Stochastic Differential Equations and Related Ordinary Differential Equations}

Let $\sigma(x)=\left(\sigma_{j}{ }^{a}(x)\right),(1 \leqq \alpha, j \leqq d)$ be a $d \times d$-matrix valued function defined on $R^{d}$. We assume that each component of $\sigma(x)$ is a bounded twice continuously differentiable function whose partial derivatives of order $\leqq 2$ are all bounded. We will consider a sequence $\left\{B_{\delta}(t)\right\}$ of approximations to $B(t)$ such that $\left\{B_{\delta_{n}}(t)\right\} \in \mathcal{A}(B ; \kappa, S)$ for some skewsymmetric $d \times d$-matrix $S=\left(s_{i j}\right) . \quad$ Let $X_{\delta}(t)=\left(X_{\hat{o}}^{1}(t), X_{\hat{0}}^{2}(t), \cdots, X_{\hat{\delta}}^{d}(t)\right)$ be the unique solution of the following ordinary differential equation:

$$
\left\{\begin{array}{l}
d X_{\delta}(t)=\sigma\left(X_{\delta}(t)\right) d B_{\delta}(t), \\
X_{\delta}(0)=x_{0} \in R^{d}
\end{array}\right.
$$

Let $X(t)=\left(X^{1}(t), X^{2}(t), \cdots, X^{d}(t)\right)$ be the unique solution of the following stochastic differential equation: 


$$
\left\{\begin{array}{l}
d X^{\alpha}(t)=\sum_{j=1}^{d} \sigma_{j}^{\alpha}(X(t)) \circ d B^{j}(t) \\
\quad+\sum_{i, j=1}^{d} \sum_{\beta=1}^{d} s_{i j}\left(\sigma_{i}^{\beta} \frac{\partial}{\partial x^{\beta}} \sigma_{j}^{\alpha}\right)(X(t)) d t, \text { for } 1 \leqq \alpha \leqq d, \\
X(0)=x_{0} \in R^{d} .
\end{array}\right.
$$

The result we want to show is the following:

Theorem 4. 1. If $\left\{B_{\delta_{n}}\right\} \in \mathcal{A}(B ; \kappa, S)$, then

$$
\lim _{n \rightarrow \infty} E_{0}\left[\left\|X_{\hat{o}_{n}}(t)-X(t)\right\|^{2}\right]=0, \quad \text { for } t \geqq 0 \text {. }
$$

Proof. The proof uses the same lemmas as in the proof of Theorem 2.1. First we note that for every $\delta>0$ and $s \geqq 0$,

$$
\left\|X_{\delta}(s)-X_{\delta}\left([s]^{-}(\delta)\right)\right\| \leqq K_{3} \sum_{m=1}^{d} \int_{[s]^{-(\delta)}}^{[s]^{+}(\delta)}\left|\dot{B}_{\delta}{ }^{m}(u)\right| d u
$$

where $K_{3}$ is a positive constant depending only on $\sigma$. By integration by parts, we have

$$
\begin{gathered}
X_{\delta}^{\alpha}\left([t]^{-}(\delta)\right)-X_{\delta}^{\alpha}(0)=\sum_{j=1}^{d} \int_{0}^{[t]^{-(\delta)}} \sigma_{j}^{\alpha}\left(X_{\delta}\left([s]^{-}(\delta)\right)\right) d B^{j}(s) \\
+\sum_{i, j, \beta=1}^{d} \int_{0}^{[t]^{-(\delta)}} \sigma_{i}^{\beta} \frac{\partial}{\partial x^{\beta}} \sigma_{j}^{\alpha}\left(X_{\delta}(s)\right) \dot{B}_{\delta}^{i}(s)\left(B_{\delta}^{j}\left([s]^{+}(\delta)\right)\right. \\
\left.-B_{\delta}^{j}(s)\right) d s, \quad \text { for } \quad \alpha=1,2, \cdots, d .
\end{gathered}
$$

Now put $c_{i j}=s_{i j}+\delta_{i, j} / 2$. Then, by (4.2),

$$
\begin{aligned}
X^{\alpha}(t)-X^{\alpha}(0) & =\sum_{j=1}^{d} \int_{0}^{t} \sigma_{j}^{\alpha}(X(s)) d B^{j}(s) \\
& +\sum_{i, \beta=1}^{d} c_{i j} \int_{0}^{t}\left(\sigma_{i}^{\beta} \frac{\partial}{\partial x^{\beta}} \alpha_{j}^{\alpha}\right)(X(s)) d s, \quad \alpha=1,2, \cdots, d .
\end{aligned}
$$

Combining this with (4.5), we have

$$
X_{\delta}{ }^{\alpha}(t)-X^{\alpha}(t)=\sum_{j=1}^{6} I_{j}^{\alpha}(t ; \delta), \quad \alpha=1,2, \cdots, d,
$$

where

$$
\begin{aligned}
& I_{1}^{\alpha}(t ; \delta)=X_{\delta}^{\alpha}(t)-X_{\delta}^{\alpha}\left([t]^{-}(\delta)\right)-X^{\alpha}(t)+X^{\alpha}\left([t]^{-}(\delta)\right), \\
& I_{2}^{\alpha}(t ; \delta)=\sum_{j=1}^{d} \int_{0}^{[t]^{-(\delta)}}\left[\sigma_{j}^{\alpha}\left(X_{\delta}\left([s]^{-}(\delta)\right)\right)-\sigma_{j}^{\alpha}(X(s))\right] d B^{j}(s),
\end{aligned}
$$




$$
\begin{aligned}
I_{3}^{\alpha}(t ; \delta)= & \sum_{i, j, \beta=1}^{d} \int_{0}^{[t]^{-(\delta)}} \sigma_{i}{ }^{\beta} \frac{\partial}{\partial x^{\beta}} \sigma_{j}^{\alpha}\left(X_{\delta}\left([s]^{-}(\delta)\right)\right) \\
& \times\left[\dot{B}_{\delta}{ }^{i}(s)\left(B_{\delta}{ }^{j}\left([s]^{+}(\delta)\right)-B_{\delta}{ }^{j}(s)\right)-c_{i j}(\delta)\right] d s, \\
I_{4}^{\alpha}(t ; \delta)= & \sum_{i, j, \beta=1}^{d} \int_{0}^{[t]^{-(\delta)}}\left[\sigma_{i}^{\beta} \frac{\partial}{\partial x^{\beta}} \sigma_{j}^{\alpha}\left(X_{\delta}(s)\right)-\sigma_{i}^{\beta} \frac{\partial}{\partial x^{\beta}} \sigma_{j}^{\alpha}\left(X_{\delta}\left([s]^{-}(\delta)\right)\right)\right] \\
& \times \dot{B}_{\delta}{ }^{i}(s)\left(B_{\delta}{ }^{j}\left([s]^{+}(\delta)\right)-B_{\delta}{ }^{j}(s)\right) d s, \\
I_{5}^{\alpha}(t ; \delta)= & \sum_{i, j, \beta=1}^{d} \int_{0}^{[t]^{-(\delta)}}\left[\sigma_{i}{ }^{\beta} \frac{\partial}{\partial x^{\beta}} \sigma_{j}^{\alpha}\left(X_{\delta}\left([s]^{-}(\delta)\right)\right)\right. \\
& \left.\quad-\sigma_{i}{ }^{\beta} \frac{\partial}{\partial x^{\beta}} \sigma_{j}^{\alpha}(X(s))\right] d s c_{i j},
\end{aligned}
$$$$
I_{\mathrm{B}}^{\alpha}(t ; \delta)=\sum_{i, j, \beta=1}^{d} \int_{0}^{[t]^{-(\delta)}} \sigma_{i}^{\beta} \frac{\partial}{\partial x^{\beta}} \sigma_{j}^{\alpha}\left(X_{\delta}\left([s]^{-}(\delta)\right)\right) d s\left[c_{i j}(\delta)-c_{i j}\right] .
$$

Now fix $T>0$. Set

$$
Z_{1}(s, \omega)=\sum_{\beta=1}^{d}\left(\sigma_{i}^{\beta} \frac{\partial}{\partial x^{\beta}} \sigma_{j}^{\alpha}\right)\left(X_{\delta}\left([s]^{-}(\delta)\right)\right) .
$$

Then $Z_{1}(s, \omega)$ is a bounded $\mathscr{F}_{s}$-adapted process with piecewise continuous sample paths. Next set

$$
Z_{2}(s, \omega)=\sum_{\beta=1}^{d}\left[\sigma_{i}{ }^{\beta} \frac{\partial}{\partial x^{\beta}} \sigma_{j}^{\alpha}\left(X_{\delta}(s)\right)-\sigma_{i}{ }^{\beta} \frac{\partial}{\partial x^{\beta}} \sigma_{j}{ }^{\alpha}\left(X_{\delta}([s]-(\delta))\right)\right] .
$$

Then, by (4.4), $Z_{2}(s, \omega)$ satisfies (3.10) in Lemma 3.4. Hence we can apply Lemma 3.3 and Lemma 3.4 to $I_{3}{ }^{\alpha}(t ; \delta)$ and $I_{4}^{\alpha}(t ; \delta)$ respectively. Hence, using (4.4) and $\left\{B_{\delta_{n}}\right\} \in \mathcal{A}(B ; \kappa, S)$, we obtain

$$
\begin{gathered}
E_{0}\left[\left\|X_{\delta_{n}}(t)-X(t)\right\|^{2}\right] \leqq K_{4} \int_{0}^{t} E_{0}\left[\left\|X_{\delta_{\eta}}(s)-X(s)\right\|^{2}\right] d s+\varepsilon_{n}, \\
\text { for } t \leqq T
\end{gathered}
$$

where $K_{4}$ is a positive constant depending only on $\sigma, \kappa$ and $T$ and $\left\{\varepsilon_{n}\right\}$ is a sequence of positive numbers with $\lim _{n \rightarrow \infty} \varepsilon_{n}=0$ depending only on $\sigma$, $\kappa$ and $T$. By (4.7), we have

$$
E_{0}\left[\left\|X_{\delta_{n}}(t)-X(t)\right\|^{2}\right] \leqq \varepsilon_{n} \exp \left(K_{t} t\right), \quad \text { for } t \leqq T,
$$

which implies (4.3). 


\section{References}

[1] Gaveau, M. B., Solutions fondamentales, représentations, et estimées sous-elliptiques pour les groupes nilpotents d'ordre 2, C. R. Acad. Sc. Paris, 282 (1976), 563-566.

[2] Itô, K., Stochastic differentials, Appl. Math. Optimization, 1 (1975), 374-381.

[3] Lévy, P., Processus stochastiques et mouvement brownien, Gauthier-Villars, Paris, 1948.

[4] McShane, E. J., Stochastic differential equations and models of random processes, Proc. 6-th Berkeley Symp. on Math. Statist. and Prob., 3 (1970), 263-294.

[5] Wong, E. and Zakai, M., On the convergence of ordinary integrals to stochastic integrals, Ann. Math. Statist., 36 (1965), 1560-1564.

[6] Wong, E. and Zakai, M., Riemann-Stieltjes approximations of stochastic integrals, Z. Wahrscheinlichkeitstheorie verw. Geb., 12 (1969), 87-97. 